\title{
The business cycle in a financially deregulated context: theory and evidence
}

\author{
Moritz Cruz ${ }^{\mathrm{a},{ }^{*}}$ \\ aUniversity of Manchester.
}

\begin{abstract}
This article proposes an amended version of Minsky's financial instability hypothesis for open, developing economies (FIH-ODE) as a theoretical underpinning for three states business cycles models. This enables a better understanding of the performance of business cycles in emerging economies during the last two decades. Applying a three states autoregressive Markov switching model to Mexico's real GDP, we investigate its business cycle behaviour and asymmetries to confirm the FIHODE hypotheses. The estimated probabilities suggest, for example, that after the financial liberalization programme was fully launched, in the late eighties, the economy shifted from the regime of medium to high growth (and vice versa) swiftly, reflecting the dependence on capital flows. The estimated parameters indicate that the average length of the business cycle has not changed, but the magnitude of expansion and crisis regimes has become more explosive and deeper, respectively.
\end{abstract}

Key Words: Latin America, Mexico, business cycles, financial liberalization, emerging economies, Autoregressive-Markov switching model.

JEL classification: E12, E32, F41, F32.

\section{Introduction}

Most authors analysing and drawing inferences regarding business cycle movements implicitly assume that the economy is operating in one of two regimes, namely expansions and contractions (see, inter alia, Hamilton, 1989 and Kim \& Nelso n, 1999). Others, however, (see, for example, Krolzig, 1997a,b and Clements \& Krolzig, 1998) have shown that a three states business cycle model generates better regime durations consonant with, for example, the NBER dated turning

\footnotetext{
* Corresponding author: School of Economic Studies, University of Manchester, Oxford Road, M13 9PL, England. Tel. +44-161-25754737, Fax +44-161-2754928.

I would like to thank to Bernard Walters and Ed Amman for their helpful comments. The usual disclaimers apply. I thank as well to CONACYT for financial support.

e-mail address: msrajac2@stud.man.ac.uk
} 
points. In this case, however, there is a shortcoming in terms of economic theory. The three states (or more regimes) assumption is supported mainly by statistical evidence. In the former case, the hypothesis of a two state business cycle model is based on theoretical definitions of the business cycle (i.e. Keynes, 1936; Burns \& Mitchell, 1946 or Hicks, 1950). In sum, the traditional theory has suggested two regimes, empirical work then has suggested three regimes, but without theoretical justification.

But Minsky's $(1975,1986)$ seminal financial instability hypothesis $(F I H)$ does provide a good theoretical explanation for three states business cycle models, and further, its development in the $\mathrm{FIH}$ for open, developing economies $(\mathrm{FIH}-\mathrm{ODE})^{1}$ facilitates application to these economies. Within this framework, for example, the role of the financial liberalization strategy as an element that influences the business cycle asymmetries and its stability, via the surge of domestic credit supply and speculative activities, respectively, is considered. That is, it is expected that once the financial deregulation strategy has been launched the average length and magnitude of the business cycle would be altered in the sense that the economy is likely to last longer in the expansion phase and contractions may be shorter and downs (ups) could be deeper (higher) than under a financially regulated situation. When the economy is in the expansion phase, the boom in speculative activities implies that there is a potential to shift from one regime to another swiftly, reflecting how vulnerable it is to these flows.

Having as a benchmark the popular Markov switching autoregressive (MSAR) model the intention of this paper is precisely to shed light on these hypotheses. Using the quarterly seasonally adjusted real GDP from 1980q1 to 
2000q4, the MS-AR model is applied to an emerging economy. We choose the Mexican economy because it sparked the new era of financial crises in emerging economies. The aim is then to show that when financial liberalization was fully implemented, Mexico's business cycle evolved as the FIH -ODE states. Section 2 provides the theoretical base for a three regimes business cycle and further explications of its evolution in a financially deregulated environment. Section 3 describes the MS-AR model methodology while section 4 discusses the results. Finally, section 5 presents the conclusions.

\section{The FIH-ODE and the three states business cycle.}

Vintage macroeconomic empirical studies searching for business cycle movements $^{2}$ (see Hamilton, 1989) have assumed the economy operates in two regimes, with the regimes associated with business cycle expansions and contractions. This general assumption basically follows the definition of the business cycle of Burns \& Mitchell (1946: 3). The MS-AR model has been applied successfully in this regard, mainly in developed economies, spotting expansions and contractions that correspond fairly closely to traditionally dated turning points published by the NBER and/or the Center of International Business Cycle Research (CIBCR) (see Krolzig, 1997a, for example). However, recent articles have shown that business cycles can exhibit more than two states. As Öcal \& Osborn (2000:27) point out "there is growing evidence that at least three regimes are required to represent adequately business cycles movements". These states have been associated with "expansions typically consisting of a period of rapid 
recovery followed by one of slower growth" (Idem). ${ }^{3}$ In fact, these sorts of models are able to date the shifts that an economy has undergone in a better fashion, though their forecast outcomes are not necessarily more accurate (see Clements \& Krolzig, 1998). However, the lack of economic theory that considers more than two regimes is a constant shortcoming in these models. The ir characterisation of a three (or more) states business cycle is based mainly on statistical evidence.

The well-known Minsky's FIH $(1975,1986)$ recognizes that the process from robustness to financial fragility involves passing through three optimisticexpectations states associated with economic expansions (namely, medium and high growth). Each regime is related to a firm's margin of safety. ${ }^{4}$ Overall, in this sense, large margins of safety will indicate the economy is in the tranquil state (medium growth), with the majority of the firms classified as hedge units. As the economy evolves, with optimistic expectations gaining ground and the investing financing process increasing as well, the margins of safety are expected to decrease; firms are categorized as speculative units, the economy is therefore classified as being in the prosperity regime (medium or high growth). Finally when the margins of safety are low, Ponzi units will predominate and the economy will be identified as being in the boom regime (high growth). The degree of financial fragility evolves in a parallel fashion and hence it is possible to predict if the economy is close to moving into the low growth regime. ${ }^{5}$

Nevertheless, in a financially deregulated context, despite the existence the above regimes, the classification of units is not so straightforward. A firm can resemble a hedge and a Ponzi unit at the same time. This is basically because, in this context, units are propelled to embark on long term gestation projects issuing 
debts repayable in foreign currency. If the unit expects to be able to meet adequately its financial commitments it can be considered, then, a hedge unit. However, the same unit can be classified as a speculative one, with a tendency to resemble a Ponzi, since it is now more vulnerable to both domestic and external financial conditions. Furthermore, a unit that “... borrowed short-term in foreign currency to finance domestic long-term assets would also be speculatively financing itself under both of Minsky's criteria: as well as needing continually to roll debts over, it will also be vulnerable to changes in interest rates... [and] to exchange-rate movements" (Arestis \& Glickman, 2002: 242). The resultant superspeculative financing unit will be the sort of unit that will predominate and therefore the regime in which the economy is operating cannot be inferred from the firms' margins of safety in a direct fashion. Consequently, the degree of financial fragility is no longer so evident either.

A first contribution then of both the seminal $\mathrm{FIH}$ and the $\mathrm{FIH}-\mathrm{ODE}$ frameworks is a sound underpinning for three states business cycle models. In this case, however, expansions typically consist of a period of mild recovery (medium growth) followed by one of explosive (high) growth. The regime of recessions is related to crisis in emerging economies. This three state model could either be able to characterise in better way business cycle turning points or simply be a useful framework that allows an explanation of the manner in which the economy evolves until it reaches the state associated with crisis.

But the FIH-ODE framework also offers a sound explanation for the sharper movements that emerging economies have registered recently in terms of growth and crises. In a liberalized economy, and assuming free mobility of capital, 
asymmetries in the business cycle can be altered considerably due to the boost to domestic credit. If investors find adequate conditions in a specific emerging economy, they will likely flood the country with inflows of capital. The following surge of domestic credit, assuming optimistic expectations gaining ground, will meant that agents will not find any obstacle to financing their projects, either in the domestic market or abroad. In the same fashion, banks will be able to increase both their loans and deposits, especially because they feel the same euphoria as businessmen. Investment and consumption as a consequence, are likely to increase considerably. Hence a larger rise in output would be expected than otherwise. The magnitude of the business cycle, in the expansion phase, can be considerably altered. The quicker and larger the inflows arrive the higher the rate of growth would be expected to be.

The duration of this phase can be altered accordingly. If the stream of flows is constant in terms of volume and periodicity, then the expansion period will likely last longer. By the same logic, constant (and large) outflows of capital will negatively affect the expansion phase, leading the economy to move quickly to the crisis regime. The magnitude of the crisis will depend essentially on the degree of existing macro imbalances. Large current account deficits coupled with high levels of banking, private and government leverage, developed during the expansion phase, will produce a sharp decrease in output if, on the one hand, the supply of credit reduces or, on the other hand, because of the economic measures that the government takes in the event of capital flight or currency attack or both. These measures will likely involve a further increase in the domestic rate of interest and the floating of the exchange rate. The length of the crisis state will depend on how 
quickly the authorities are able to regain investors' confidence if they decide to continue applying the liberalization strategy or on the speed with which the international community is able to provide a financial bailout.

Finally, the FIH-ODE approach is able to provide us with the logic to explain the instability that the business cycle during the upswing can undergo, which is reflected in swift regime shifts. As the economy becomes more financially liberalised, speculative activities become larger and larger because these transactions validate their profits through the increase in assets prices. If success breeds success, the economy will move to the high growth regime (and will continue operating there) as long as the foreign investments validate their profits. The pace and stability of the economy will indeed be dependent on these inflows, because agents' expectations will be based mainly on the returns from these activities and the stream of these flows may have the power to dictate the stability of key variables, such as the domestic rate of interest and the exchange parity. A sudden decrease in inflows of foreign capital, for example, would be expected to have a negative impact on the foreign exchange rate alongside a rise in the domestic rate of interest. In the very short term, even when the trend of foreign capital inflows returns to its former level or ceases to flee, a negative shift in agents' expectations might be expected to gain ground because their financial commitments might have been increased considerably, as well as current and future income flows which could also be affected negatively. ${ }^{6}$ As a consequence, the regime in which the economy is currently operating has the potential to change dramatically, i.e. from the high to the medium growth regime. In other words, the evolution of the business cycle would likely reflect this effect, moving swiftly from 
one regime to another. But it could operate for very short periods in one regime and then shift to another regime and again change to another, with the associated result that the economy may be seen operating in two regimes at the same time.

\section{Markov switching models.}

MS-AR models are based in the following idea. Consider a stationary time series $\left\{y_{t}\right\}$ that is assumed to have been generated by an $\operatorname{AR}(p)$ process and is influenced in its mean $(\mu)$ by a unobserved random variable $s_{t}$, which can be called the state or regime $(M)$ that the process was in at date $t$. To keep exposition as simply as possible consider an $\mathrm{AR}(1)$ process with only two states. ${ }^{7}$ Following Krolzig's (1997a) nomenclature, this process can be labelled an MSMean(2)-AR(1) process,

$y_{t}-\mu_{s_{t}}=\phi\left(y_{t-1}-\mu_{s_{t-1}}\right)+\varepsilon_{t}, \quad$ with $\varepsilon_{t} \sim N I D\left(0, \sigma^{2}\right)$

If the $s_{t}=1$, then the process is in regime one and $\mu_{1}$, while $s_{t}=2$ means the process is in regime 2 and $\mu_{2}$.

In the business cycle context, for example, if $\left\{y_{t}\right\}$ represents output growth of the economy, then we might have $\mu_{1}$ corresponding to periods of recession and $\mu_{2}$ corresponding to periods of expansion.

An alternative possibility to equation (1) is

$y_{t}=\delta_{s_{t}}+\phi y_{t-1}+\varepsilon_{t}$

in this case the intercept shifts with the regime. ${ }^{8}$ The process can be labelled then as an MSIntercept $(M)-A R(p)$ model. In practice both equations give similar results. 
However, "for a MSI model smoothing and filtering probabilities are less computationally demanding (and therefore much faster) than the statistical analysis with a MSM model. Hence, if there are not theoretical reasons which call for an MSM specification, an MSI specification is preferred" (Krolzig, 1997a:126).

On the other hand, it is possible as well that for an MSM or MSI specification the variance changes with the regime. This allows the following specification

$$
\begin{array}{ll}
y_{t}=\delta_{1}+\phi y_{t-1}+\varepsilon_{t}, & \varepsilon_{t} \sim N I D\left(0, \sigma_{1}^{2}\right) \text { when } \mathrm{s}_{\mathrm{t}}=1 \\
y_{t}=\delta_{2}+\phi y_{t-1}+\varepsilon_{t}, & \varepsilon_{t} \sim N I D\left(0, \sigma_{2}^{2}\right) \text { when } \mathrm{s}_{\mathrm{t}}=2
\end{array}
$$

In this case the process is labelled as an MSIH(2)-AR(1).

Markov chains are the mechanism that govern the evolution of the stochasic and unobservable regimes on which the parameters of the autoregression depend (Clements \& Krolzig, 1998: 55).

For a first order Markov chain the probability the $s_{t}$ equals some particular value $j$ depends on the past only through the most recent value $s_{t-1}$ :

$P\left\{s_{t}=j \mid s_{t-1}=i\right\}=p_{i j}$

Such a process is described as an $\mathrm{N}$-state Markov chain with transition probabilities $\left\{p_{i j}\right\}_{i j=1,2, \ldots N}$. The transition probabilities $p_{i j}$ give the probability that state $i$ will be followed by state $j$. Note that $\sum_{j=1}^{N} p_{i j}=1$.

For a two-state Markov chain, the time-invariant transition probabilities matrix is:

$\mathbf{P}=\left[\begin{array}{ll}p_{11} & p_{21} \\ p_{12} & p_{22}\end{array}\right]$ 
Row one column two, for example, gives the probability that state 2 will be followed by state 1. The matrix $\mathbf{P}$ is ergodic, irreducible and does not exhibit an absorbing state (see Hamilton, 1994: Ch. 22).

To estimate the vector of parameters $\Phi=\left(\mu_{s}, \phi_{1}, \sigma^{2}, p_{11}, p_{22}\right)$, the likelihood function of the MSM(2)-AR(1) model is maximized through an iterative estimation technique. The maximum likelihood (ML) estimation of the model is based on an implementation of the expectation maximization (EM) two steps algorithm proposed by Hamilton (1989). ${ }^{9}$ The expectation step involves a pass through the filtering and smoothing algorithms, using the estimated parameter vector $\Phi^{(j-1)}$ of the last maximization step in place of the unknown true parameter vector. This delivers an estimate of the smoothed probabilities $P\left(S \mid Y, \Phi^{(j-1)}\right)$ of the unobserved states. In the maximization step, an estimate of the parameter vector $\Phi$ is derived as a solution $\Phi$ of the first order conditions associated with the likelihood function, where the conditional regime probabilities $P(S \mid Y, \Phi)$ are replaced with the smoothed probabilities $P\left(S \mid Y, \Phi^{(j-1)}\right)$ derived in the previous expectation step. Equipped with the new parameter vector $\Phi$ the filtered and smoothed probabilities are updated in the next expectation step, and so on, guaranteeing an increase in the value of the likelihood function at each step (Clements \& Krolzig, 1998: 56). ${ }^{10}$

\section{The results}

The sample period of Mexico's quarterly real seasonally adjusted GDP is $1980 \mathrm{q} 1$ to $2000 \mathrm{q} 4 .^{11}$ Using the rate of growth of this variable ${ }^{12}$ to ensure 
stationary, ${ }^{13}$ we found that an adequate "three states business cycle" model (in the sense of generating an evolution consonant with the FIH-ODE framework applied to the Mexican 1994-95 experience) is an $\mathrm{MSIH}(3)-\mathrm{AR}(4)$, the flag $\mathrm{H}$ stands for the heteroskedastic term: ${ }^{14}$

$y_{t}=\delta_{s_{i}}+\sum_{k=1}^{4} \phi_{k} y_{t-k}+\varepsilon_{t}$

where $\varepsilon_{t} \sim N I D\left(\sigma^{2}\left(s_{t}\right)\right)$ and $s_{t} \in\{1,2,3\}$ is generated by a Markov chain. The specification has a shifting intercept term (MShtercept). The number of lags is four. ${ }^{15}$

Figure 1 and table 1 summarize the business characteristics of this model. ${ }^{16}$ The figure depicts the filtered and smoothed probabilities of the crisis regime "C", the medium, "M", and high growth, "H", regimes, with the last two regimes associated with the expansion phase described in the FIH-ODE framework.

Figure 1 Smoothed and filtered probabilities of regimes $\mathbf{C}, \mathbf{M}$ and $\mathrm{H}$

Immediately after a period of economic instability from the mid 1980s, there was a substantial recovery in the economy. Both smoothed and filtered probabilities confirm this fact. As can be seen, from early 1987 the economy is already operating in regime $\mathrm{M}$, and although decreasing, the probability of operating in this regime is high over the next two years. ${ }^{17}$ It is precisely in late 1988 when the economy starts to shift to the state H. Moreover it is in late 1989 when the probability of the economy operating in state $\mathrm{H}$ starts to get close to one. In fact, the negligible probability of operating in state M in 1990 contrasts clearly with 
the almost unitary probability of regime $\mathrm{H}$. It is important to note, then, that the probability of both regimes is practically reversed between 1987 and 1990. This empirical evidence allows the inference that Mexico's business cycle regimes initially evolved as the classical Minsky's FIH states (a mild recovery followed by explosive growth).

Financial deregulation strategy initiated from 1977 and was intensified in 1988, but it can be argued that in 1990 fully financial openness was fully launched, ${ }^{18}$ coupled with the trade liberalization strategy (for example, the announcement of the Mexico-USA trade agreement took place in 1990). ${ }^{19}$

On the other hand, the evolution and behaviour of the business cycle regimes $\mathrm{M}$ and $\mathrm{H}$ during the period from early 1991 to late 1994 seems to be consistent with the FIH-ODE, in the sense that once the economy was totally opened the business cycle reflected the increased exposure to domestic and external market conditions, especially due to capital flows. First of all, and despite the probabilities of regime $\mathrm{M}$ and $\mathrm{H}$ maintaining an inverse evolution during early $90 \mathrm{~s}$, it is difficult to categorize the regime in which the economy is operating in a straightforward fashion. In fact, from 1991q1 to $1991 \mathrm{q} 4$ the economy operates in regime $M$ with an average probability of 0.5 , but in 1991 q3 it could be argued that it is operating in regime $\mathrm{H}$ as well. The next year exhibits a similar pattern. For example, the probability of operating in regime $\mathrm{H}$ from $1992 \mathrm{q} 1$ to $1992 \mathrm{q} 3$ is around 0.6. However, from late 1992 to late 1993, the economy is already operating in the $M$ state, then swiftly shifts to regime $H$, stays there for a very short period (only one quarter) and then shifts again dramatically to regime $\mathrm{M}$. 
Table 1 Parameters Estimates for MSIH(3)-AR(4) of Mexico's GDP, 1980q1-2000q4 (Standard Errors in Parenthesis)

In sum the economy operates indistinctly in either one of the regimes or in both at the same time. This point is also consistent with our framework. Due to the financially liberalized context, as stated earlier, the economy is now more exposed to external and internal shocks, its business cycle exhibits, then, dramatic shifts which are reflected in the regimes in which the economy is operating. This remarkable evidence seems to be supported by the $\mathrm{MSIH}(3)-\mathrm{AR}(4)$ model test for asymmetries (see table 2). ${ }^{20}$ The model does not reject the hypothesis of nonsharpness, there is clear evidence of symmetric turning points (or sharpness). The probability, then, of moving from one regime to another is equal.

\section{Table 2 Test for Asymmetries}

The macroeconomic fluctuations in Mexico during the last twenty years are marked by three major depressions, associated more precisely with periods of crisis. In the early 80 s, the first one, the well-known debt crisis of 1982 , began in the first quarter of 1982 and ended approximately in the second quarter of 1983. A period of negative growth followed, associated with a world recession, from late 1986 to late 1987 . Finally, the shorter but pronounced Peso crisis occurred, which, howe ver, only lasted the first two quarters of 1995 (see figure 2).

Figure 2 Mexico real rate of GDP-growth, year over year 
Importantly, although not surprisingly, during the period of the Peso crisis, late 1994 until mid 1995, the probability of both regimes, $\mathrm{M}$ and $\mathrm{H}$, coincides for the very first time. However, and in contrast with previous crises, the economy operates in regime $\mathrm{C}$ for very short time. This fact might be explained on the basis of the series of economic announcements and policy measures that the authorities took in the aftermath of the crisis (inter alia, but mainly, the reinforcement of mainstream economic policies aimed at continuing the openness strategy, the USA financial bailout package and the creation of a sort of federal insurance saving fund $\left.^{21}\right)$. In fact, the recovery of agents' confidence, coupled with the end of the financial panic seems to have started from May $1995 .{ }^{22}$ As is to be expected the economy, after the 1994-95 collapse, starts to operate in regime M.

In this sense, the $\mathrm{MSIH}(3)-\mathrm{AR}(4)$ model is a model that fairly closely corresponds to the periods of crisis more than dated business cycle turning points. ${ }^{23}$ The CIBCR methodology, for example, registers two additional recessions, one in 1988 and the other in 1993, meanwhile the methodology of Rand \& Tarp (2002) ${ }^{24}$ adds another recession (1991) though it does not report the 1994-95 crisis (see table 3). But figure 3 shows that these are clearly not reflected in the quarterly GDP growth rate of these periods, and hence not detected by the model. ${ }^{25}$ In other words, the smoothed and filtered probabilities of regime $\mathrm{C}$ remain negligible during the period of interest. This result further supports the hypothesis that the conditions for the crisis originated during this period in the sense that he economy was always operating in either one of the other two regimes associated with the expansions, but never in the state $C .{ }^{26}$ 
Table 3 Business cycle chronologies, 1980-2000

Figure 3 Mexico real rate of GDP-growth, quarter over quarter and contribution of the Markov chain to the business cycle

The estimated parameters given in table 1 shed light on the asymmetries of the business cycle. First of all, the average duration of the business cycle (12.7 quarters) is in line with the average length (12 quarters) for emerging economies business cycles length reported by Rand \& Tarp (2002). Moreover, compared with the average business cycle length for the USA, five years, (see Clements \& Krolzig, 1998) our outcome is also consistent with Rand \& Tarp's main argument regarding the average business cycle length for transition economies. They argue that the average business cycle length for these countries is shorter than for developed economies.

Furthermore, these authors found that, among regions, for Latin America "the average length of the expansion periods is longer than the contraction period, whereas the opposite is characteristic for Asian and North Africa countries in the sample" (p. 2074). As we can see, this holds for Mexico's business cycle model as well. The average duration of the expansion phase, the $M$ and $H$ regimes together, is higher than the duration of the $C$ regime (4.6 quarters vs 3.5 quarters). Importantly, once the economy operates in regime $\mathrm{H}$, it remains there for almost six quarters, and is associated with a rate of growth of $2.1 \%$. Meanwhile, the rate of growth associated with the regime $\mathrm{C}$ is $-1.2 \%$.

This evidence suggest that, on the one hand, Mexico's business cycles have become explosive, undergoing high rates of growth or deep crises, and, on the other hand, the length of time that the economy operates in the $\mathrm{H}$ and $\mathrm{C}$ 
regimes could be effectively associated with the flows of foreign capital. Thus the economy has become more unstable.

\section{Conclusions}

This paper has proposed a further development of Minsky's $\mathrm{FIH}$, that is a version for open, developing economies, as a theoretical basis for analysing and drawing inferences from a three states business cycle model, trying to fill the theoretical gap existing in this literature.

This framework also offers further explanations for some issues relating to the evolution of the business cycle in these countries. One issue concerns the role of the financial liberalization strategy as an element that has influenced the asymmetries of the business cycle and its stability, via the surge of domestic credit supply and speculative activities, respectively. Another issue is the identification of the states in which the economy is operating and its degree of financial fragility.

To shed light on these issues, an autoregressive Markov switching model was applied to the real quarterly seasonally adjusted GDP of Mexico from 1980 to 2000. The results of the MSI(3)-AR(4) supported the view that the evolution of Mexico's business cycle is better analysed using a three states model. Furthermore, the estimated probabilities confirm the assumption that the Mexican economy in its transition from robustness to financial fragility necessarily operated in the medium and high growth regimes, in the sense that the probabilities during the period 1988-94 are negligible for the $\mathrm{C}$ regime. Moreover, the analysis of the estimated probabilities of regime $\mathrm{M}$ and $\mathrm{H}$ also indicate that after the total 
implementation of the financial liberalization strategy the economy shifted quickly from one regime to another; further asymmetric tests support the argument that the probability of shifting from one regime to another was equal. This fact makes more difficult an accurate identification of the regime in which the economy was operating as well as the detection of its degree of financial fragility.

The estimated parameters, on the other hand, suggest that the financial liberalization strategy has altered the asymmetries of Mexico's business cycle. Concretely, the magnitude of the $\mathrm{H}$ and $\mathrm{C}$ regime is more pronounced, and their duration has been altered, to larger and shorter periods of time, respectively; although the overall length of the business cycle has remained within the standards. This evidence corroborates the FIH-ODE hypotheses regarding the business cycle in emerging economies. 


\section{Notes}

${ }_{2}^{1}$ See Arestis \& Glickman (2002) and Cruz (2003).

2 Vintage in the sense that the MS-AR model assumes time-invariant transition probabilities. That is the probability of switching from one regime to another is constant over the time. A step forward in the analysis of business cycles asymmetries are the modern time-varying transition probabilities MS-AR models. In this case the probabilities of switching may be dependent on some underlying economic fundamentals (see Diebold, Lee \& Weinbanch, 1994 and Filardo, 1994).

${ }^{3}$ In the same line is Sichel (1994).

4 The margin of safety represents a cushion which absorbs any unforeseen changes in the cash inflows and outflows (Kregel, 2001: 196).

${ }^{5}$ In this sense, a crisis will necessarily arise after the economy has operated in the medium and high growth regimes. This in contrast with the conventional wisdom that proposes that a crisis may arise at any point (arguing corruption, government mismanagement, cronyism, among others, as the main cause), regardless of the regime in which the economy could be operating.

${ }^{6}$ Aggregate demand might decrease as a consequence of fiscal retrenchment and a tight monetary policy applied in order to regain investors' confidence.

${ }_{8}^{7}$ However, an extension of the procedure to the general $\operatorname{AR}(k)$ case with $N$ states would be straightforward.

${ }^{8}$ For example "In the Hamilton (1989) model there is an immediate one-time jump in the process mean after a change in the regime. It may be plausible to assume that the expected growth rate smoothly approaches a new level after the transition from one sate of the business cycle to another. For these situations, the MSI-AR model may be used" (Krolzig, 1997a: 232).

${ }^{9}$ For a more detailed explanation of the EM-algorithm refer also to, inter alia, Hamilton (1990 and 1994) and Krolzig (1997a, Ch. 6 and 9).

${ }^{10}$ It is important to notice that a widespread use of the Markov switching models has been the analysis of a set of series that follow a common or contemporaneous regime (see Krolzig, 1997a,b and Coe, 2002). These Markov switching models, labelled as $\operatorname{MS}(M)-\operatorname{VAR}(p)$, generalize the data generation process described in equations (1) and (2) to a multivariate context. Conditional on the state process, the $N$-dimensional vector of stationary time series $\left\{y_{t}\right\}$, is generated by a vector autoregression or order $p$. The $\operatorname{MSM}(2) \operatorname{VAR}(1)$, thus, would look like, $y_{t}-\mu_{s_{t}}=\mathbf{A}_{1}\left(\mathbf{y}_{\mathrm{t}-1}-\mu_{\mathrm{st}-1}\right)+\mathbf{e}_{\mathrm{t}}$ with $\varepsilon_{t} \sim N I D(0, \Sigma)$. We can also assume that the variancecovariance matrix S may be state dependent, namely $\varepsilon_{t} \sim N I D\left(0, \Sigma_{s_{t}}\right)$. Thus we have an $\operatorname{MSH}(M)-\operatorname{VAR}(p)$ process, where the mean (or the intercept) and the variance change when the regime of the process changes (Mejia-Reyes, 2000:81). The mechanism to estimate the vector of parameters $\Phi$ is basically the same as for an MS-AR model.

${ }^{11}$ The database source is the IMF- International Financial Statistics (2002), Cd-Rom.

${ }_{12}^{12}$ Quarterly growth rate is calculated as $100 * \ln \left(\mathrm{y}_{\mathrm{t}} / \mathrm{y}_{\mathrm{t}-1}\right)$.

13 The ADF statistic test for the test of the null hypothesis that a series is I(1) against the alternative that it is stationary around a constant is -4.048 for the growth rate of the seasonally adjusted real GDP. This implies a rejection of the series follows a random walk, at the 1 percent level.

${ }^{14}$ A basic way to dismiss a linear model in favour of a non-linear one is just by looking at the graph of the variable. For Mexico's real quarterly seasonally adjusted GDP figure (not presented here) we can observe in general an upward trend, which at three marked times is disturbed by large downward shifts, which display a substantial negative trend. This relative decrease in output during recession seems larger in an absolute sense than the average increase in output during expansions. It can be said, then, that Mexico GDP is asymmetric over the business cycle, and that negative shocks have a larger impact in an absolute sense than have positive shocks (see Franses, 1998, ch. 8).

${ }^{15}$ I used the Schwartz Information Criteria (SIC) to select the lag order. Despite the AR with three lags giving a lower SIC, it does not exhibit business cycle features. For example, the estimated $p_{11}$ and $p_{33}$ are only 0.38 and 0.64 , which directly translates into a very short duration of the crisis $(\mathrm{C})$ and high growth $(\mathrm{H})$ regimes. Thus for this period the MSIH(3)-AR(3) model attributes isolated observations to regimes $\mathrm{C}$ and $\mathrm{H}$ and is more a model of 'outliers' than a business cycle model. So, I chose the AR with four lags because it provides a more adequate characterization of the business cycle, and it still gives a lower SIC over the one with five lags. 
${ }_{17}$ The estimations reported herein were carried with the MSVAR class for Ox (see Krolzig, 1998).

17 Actually one would expect that the economy operate in regime $M$ soon after the official launch of the stabilization programme, in late 1988. However, it is worth recalling that some policy measures aimed at stabilizing the economy were taken at the beginning of that year.

${ }^{18}$ For example, in March 1989 the Brady Plan to refinance the external debt was announced and in July it was signed; a succession of measures relaxed bank's reserve requirements, credit quotas to high priority sectors and control in interest rates were implemented, the elimination of restrictions to foreign investment in domestic bonds, largely government bonds, and stock marke ts took place in 1989 and 1990. In order to give security to investors, the Financial Group Law was announced and passed in July 1990. The law allowed private-sector majority ownership of Mexican banks and initiated the privatisation process. Foreign investment was permitted up to $30 \%$ (Ros, 2001).

${ }^{19}$ Indeed, looking at the period 1980-86, when the financial liberalisation programme was still insignificant, it is clear that after the crises of 1982 and 1986-87 the economy operates basically only in one regime, in this case the $\mathrm{M}$ regime.

${ }^{20}$ For a detailed exposition of these tests see Clements \& Krolzig (2003).

21 This insurance was known as the FOBAPROA (Fondo Bancario de Proteccion al Ahorro).

${ }^{22}$ Investigating the timing of the crisis, following Coe's (2002) approach, we applied an MSIH(2)-VAR(9) model with the cost of credit intermediation and the growth rate of the currency-deposit ratio as variables. We found that the estimated probabilities suggest that the financial panic started in November of 1994 with the currency attack registered that month. This result supports our view that a financial crisis is usually signalled by a currency attack (see Cruz, 2003). Furthermore, the probabilities also suggest that the crisis ended (or at least the return of confidence to the markets began) in May of 1995, when the exchange rate was stabilised and the domestic rate of interest, though high, started to show a decreasing tendency (the estimated probabilities can be obtained with the author upon request).

${ }^{23}$ In fact, again, the tests for asymmetries support this view. For example, both, the model based (MB) and the non-parametric (NP) test indicates negative skewness of output growth (i.e. deepness of contractions) with the NP test permitting rejection of the null at the 5\% level. Additionally, the NP skewness test indicates steepness of recessions, but only at the $20 \%$ level of significance (see table 2).

24 These authors apply the Bry \& Boschan (1971) methodology, but using the Hodrick-Prescott and the banpass filters, to determine business cycles turning points. It is important to stress that they use the quarterly index of industrial production.

${ }^{25}$ Our results, nevertheless, are in line with the estimates of Mejia-Reyes (2000, Ch 3). Using the rate of growth of Mexico's annual income per capita for the sample period 1950-95, and applying an MSH(2)-AR(1) model, he found that the filtered probabilities correspond closely to the dates of crises highlighted above. It is important to stress that he uses the so-called AKO methodology (see Artis, Kontolemis \& Osborn, 1997) to date business cycle turning points.

${ }^{26}$ To further support this argument, we estimated an MSIH(2)-AR(5) model which exhibits exactly the same turning points in the regime $\mathrm{C}$. That is, the smoothed and filtered probabilities of regime $\mathrm{C}$ are practically negligible from 1988 until late 1994. 


\section{Graphs and tables}

Figure 1 Smoothed and filtered probabilities of regimes $\mathrm{C}, \mathrm{M}$ and $\mathrm{H}$

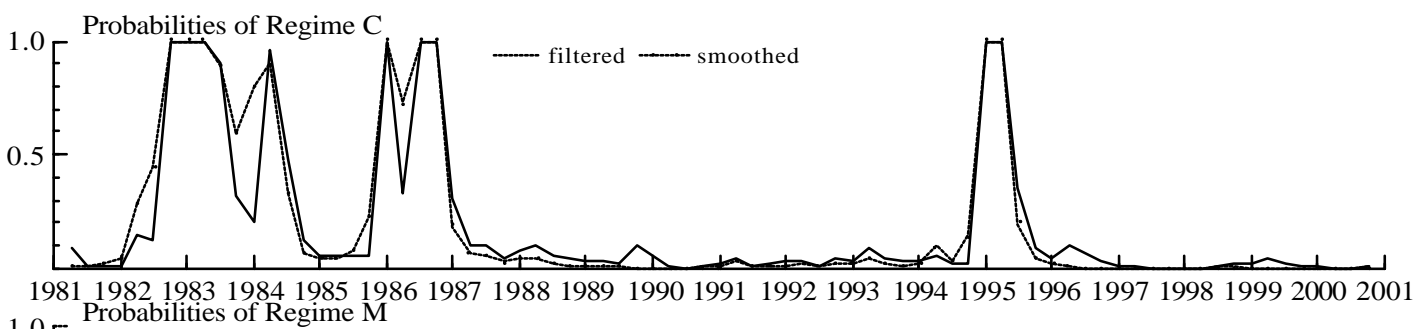
1.0 .. Probabilities of Regime M

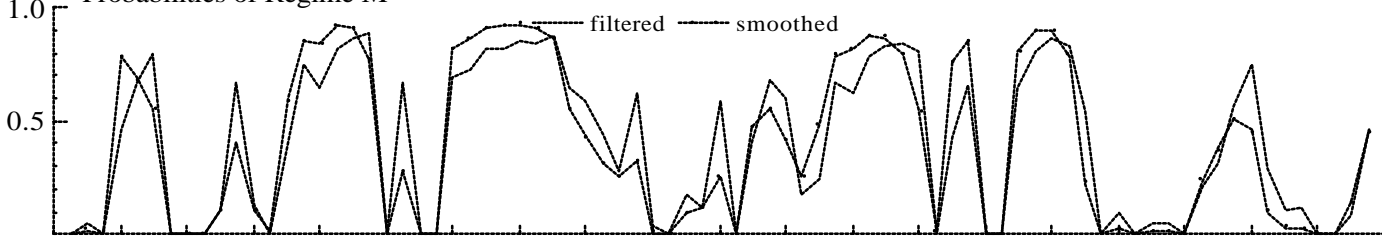

19811982198319841985198619871988198919901991199219931994199519961997199819992000200
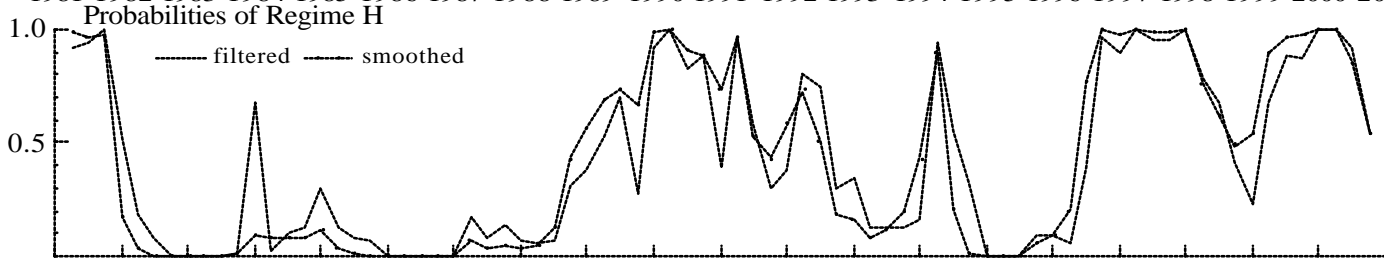

198119821983198419851986198719881989199019911992199319941995199619971998199920002001

Figure 2 Mexico real GDP-growth, year over year, 1981q1-2000q4

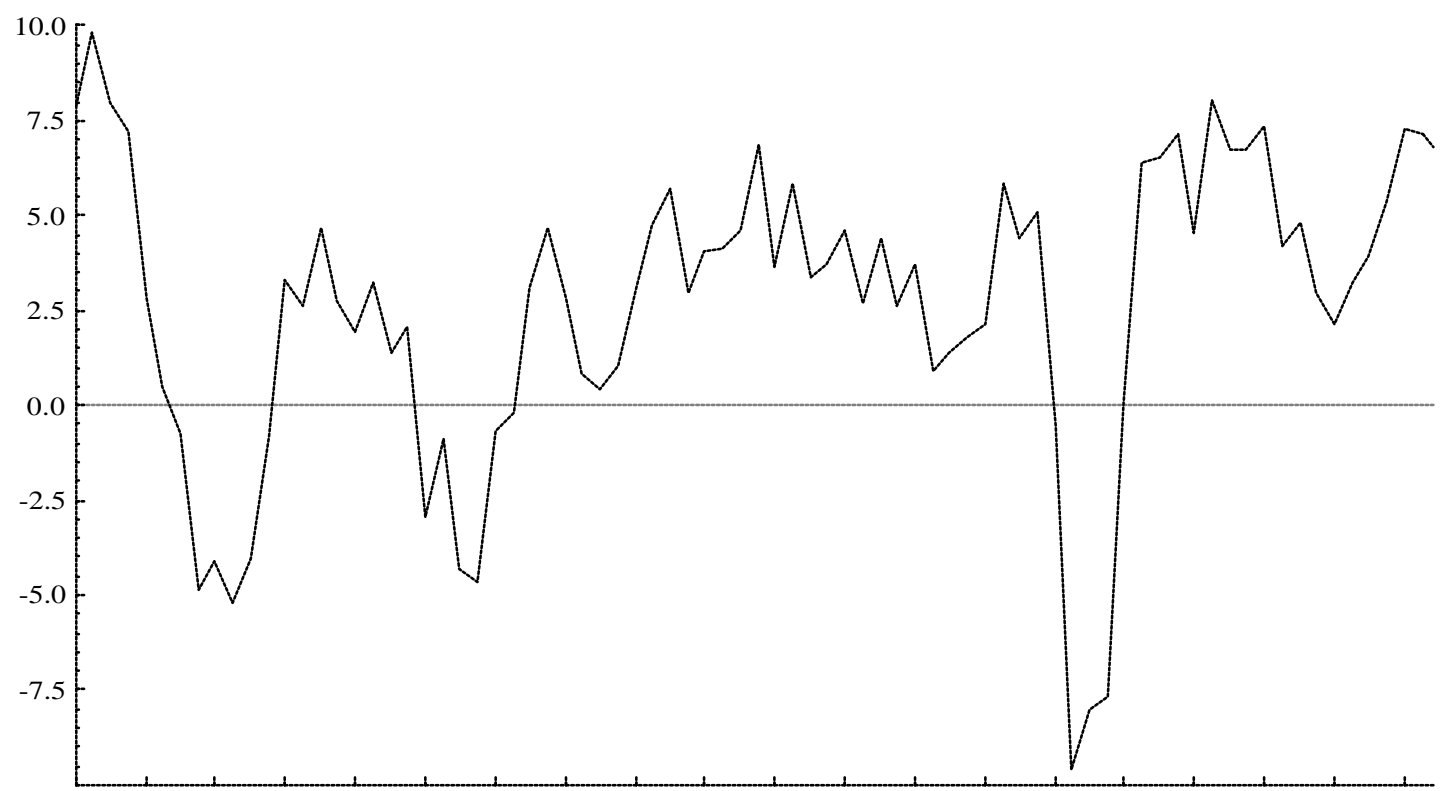

19811982198319841985198619871988198919901991199219931994199519961997199819992000 
Figure 3 Mexico real GDP-growth, quarter over quarter and contribution of the Markov chain mean to the business cycle

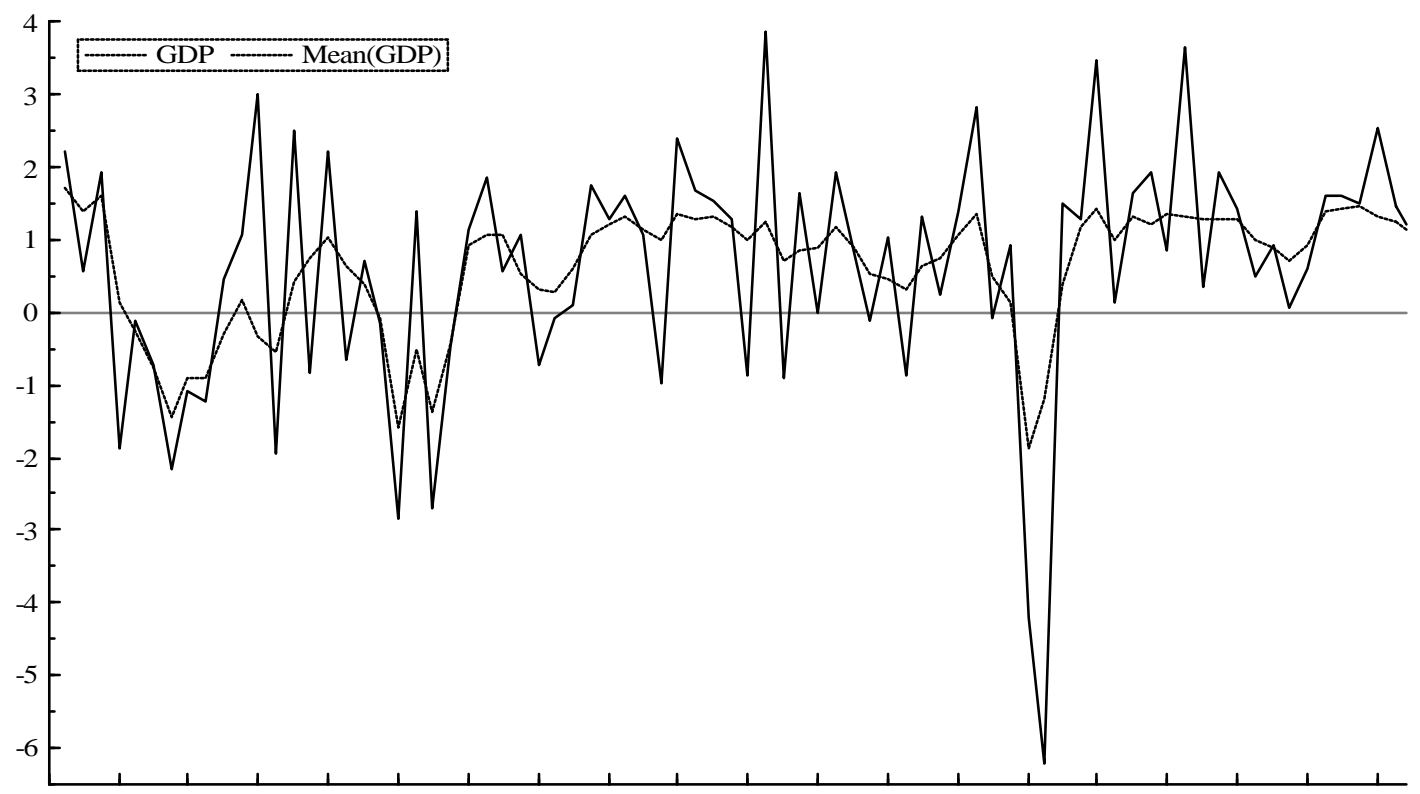

19811982198319841985198619871988198919901991199219931994199519961997199819992000 
Table 1. Parameters Estimates for $\mathrm{MSI}(3)$ -

AR(4) of Mexico's GDP, 1980q1-2000q4

(Standard Errors in Parenthesis)

\begin{tabular}{|c|c|}
\hline Parameter & Estimate \\
\hline$\delta_{C}$ & $\begin{array}{l}-1.2571 \\
(0.8142)\end{array}$ \\
\hline$\delta_{M}$ & $\begin{array}{c}0.9007 \\
(0.2246)\end{array}$ \\
\hline$\delta_{H}$ & $\begin{array}{c}2.1479 \\
(0.4684)\end{array}$ \\
\hline$\phi_{1}$ & $\begin{array}{l}-0.2634 \\
(0.0745)\end{array}$ \\
\hline$\phi_{2}$ & $\begin{array}{c}0.1246 \\
(0.0645)\end{array}$ \\
\hline$\phi_{3}$ & $\begin{array}{l}-0.3099 \\
(0.0693)\end{array}$ \\
\hline & -0.2007 \\
\hline$\phi_{4}$ & $(0.0670)$ \\
\hline$\sigma_{C}^{2}$ & 5.0149 \\
\hline$\sigma_{M}^{2}$ & 0.40899 \\
\hline$\sigma_{H}^{2}$ & 1.1007 \\
\hline Trans. Prob. $p_{c c}$ & 0.7157 \\
\hline Trans. Prob. $p_{C M}$ & 0.1350 \\
\hline Trans. Prob. $p_{C H}$ & 0.0019 \\
\hline Trans. Prob. $p_{M M}$ & 0.6916 \\
\hline Trans. Prob. $p_{M C}$ & 0.2827 \\
\hline Trans. Prob. $p_{M H}$ & 0.1654 \\
\hline Trans. Prob. $p_{H H}$ & 0.8328 \\
\hline Trans. Prob. $p_{H M}$ & 0.0016 \\
\hline Trans. Prob. $p_{H C}$ & 0.1735 \\
\hline Duration C & 3.52 \\
\hline Duration M & 3.24 \\
\hline Duration $\mathrm{H}$ & 5.98 \\
\hline Obs. in $C$ & 14.8 \\
\hline Obs. in M & 30.9 \\
\hline Obs. in $H$ & 33.3 \\
\hline SIC & 4.3383 \\
\hline Log likelihood & -136.4084 \\
\hline
\end{tabular}


Table 2. Test for Asymmetries

\begin{tabular}{|l|c|c|c|}
\hline \hline \multicolumn{1}{|c|}{ Test } & Sign & Test statistic value & $p$ value \\
\hline MS: Sharpness & & 0.63 & {$[0.88]$} \\
\hline $\mathrm{p}_{12}=\mathrm{p}_{32}$ & & 0.18 & {$[0.66]$} \\
\hline $\mathrm{p}_{13}=\mathrm{p}_{31}$ & & 0.00 & {$[0.99]$} \\
\hline $\mathrm{p}_{21}=\mathrm{p}_{23}$ & & 0.06 & {$[0.80]$} \\
\hline MS: Deepness & - & 0.37 & {$[0.53]$} \\
\hline MS: Steepness & + & 0.63 & {$[0.42]$} \\
\hline NP: Deepness & - & 17.2 & {$[0.00]^{\star \star}$} \\
\hline NP: Steepness & - & 1.8746 & {$[0.17]$} \\
\hline
\end{tabular}

Notes: The NP and MS test statistics are $?^{2}(1)$ under the null of symmetry. A positive (negative) value of "Sign" flags positive (negative) skewenss.

** Significance at the 5\% level.

Table 3. Business cycle chronologies, 1980-2000

\begin{tabular}{|c|c|c|c|}
\hline \hline \multicolumn{2}{|c|}{ CIBCR Methodology } & \multicolumn{2}{c|}{ Rand \& Tarp Methodology ${ }^{* *}$} \\
\hline Peak & Trough & Peak & Trough \\
\hline & Jan-83 & $81 . q 3$ & $83 . q 1$ \\
\hline Jan-85 & Aug-86 & $85 . q 3$ & $86 . q 3$ \\
\hline Nov-87 & Jul-88 & 87.93 & $88 . q 1$ \\
\hline Aug-89 & Aug-93 & $88 . q 4$ & $89 . q 3$ \\
\hline Jun-94 & Apr-95 & 90.94 & $91 . q 3$ \\
\hline Jun-97 & Jun-99 & $92 . q 2$ & $93 . q 1$ \\
\hline Jun-00 & & $93 . q 4$ & $94 . q 3$ \\
\hline
\end{tabular}

Source: Available at www.businesscycle.com (accessed 23 July 2003).

${ }^{* *}$ Rand \& Tarp (2002: 2075). 


\section{References}

Arestis, P. \& Glickman, M. (2002) "Financial crisis in Southeast Asia: dispelling illusion in the Minskyan way", Cambridge Journal of Economics, 26, 237-60.

Artis, M, Kontolemis, Z \& Osborn, D. (1997) "Business cycles for G7 and European countries", Journal of Business, 70, 2, 249-78.

Bry, G. \& Boschan, C. (1971) Cyclical analysis of time series: selected procedures and computer programs, Cambridge, USA.

Burns, A. \& Mitchell, W. (1946) Measuring business cycles, Cambridge, USA.

Clements, M. \& Krolzig, HM. (1998) "A comparison of the forecast performance of Markovswitching and the threshold autoregressive models of US GNP", Econometrics Journal, 1, C47C75.

Clements, M. \& Krolzig, HM. (2003) "Business cycle asymmetries: characterization and testing based on Markov-switching Autoregressions, Journal of Business \& Economic Statistics, 21, 1, 196-211.

Coe, P. (2002) "Financial crisis and the great depression: a regime switching approach", Journal of Money, Credit and Banking, 34, 1, 76-93.

Cruz, M. (2003) "A Minskyian crisis: an application to the 1994-95 Mexican experience", Discussion Paper Series 0325, School of Economic Studies, The University of Manchester.

Diebold, F., Lee, J. \& Weinbanch, G. (1994), "Regime switching with time-varying transition probabilities", in Hargreaves, C, ed., Nonstationarity time series analysis and cointegration, Oxford University Press, 283-302.

Filardo, A. (1994) "Business cycles phases and their transitional dynamics", Journal of Business \& Economic Statistics, 12, 299-308.

Franses, H. (1998) Time series models for business and economic forecasting, Cambridge University Press, England.

Hamilton, J. (1989) "A new approach to the economic analysis of nonstationary time series and the business cycle", Econometrica, 52, 2, 357-384.

Hamilton, J. (1990) "Analysis of time series subject to changes in regime", Journal of Econometrics, 45, 39-70.

Hamilton, J. (1994) Time series analysis, Princeton, USA.

Hicks, J. (1950) A contribution to the theory of economic trade, Oxford University Press, England.

IMF- International Financial Statistics (2002), Cd-Rom.

Keynes, J. (1936) The general theory of employment, interest and money, Macmillan, UK.

Kim, C. \& Nelson, C. (1999) State-space models with regime switching. Classical and GibbsSampling approaches with applications, MIT, USA.

Kregel, J. (2001) "Yes 'it' did happen again. The Minsky crisis in Asia" in Bellofiore, R. \& Ferri, P., eds., Financial fragility and the investment in the capitalist economy. The legacy of Hyman Minsky, Vol. II, Edward Elgar, UK, 195-212.

Krolzig, HM. (1997a) Markov-switching Vector Autoregressions. Modeling Statistical Inference, an application to the business cycle analysis, Lecture Notes in Economics and Mathematical Systems 454, Springer-Verlag, Berlin.

Krolzig, HM. (1997b) "International business cycles: regime shifts in the stochastic process of economic growth", Applied Economics Discussion Paper 194, Institute of Economics and Statistics and Nuffield College, Oxford University.

Krolzig, HM. (1998) "Econometric modelling of Markov-Switching Vector Autoregressions using MSVAR for Ox", Institute of Economics and Statistics and Nuffield College, Oxford University.

Mejia-Reyes, P. (2000) Essays on business cycles in Latin America, PhD Thesis, University of Manchester.

Minsky, H. (1975) John Maynard Keynes, Columbia University Press, USA.

Minsky, H. (1986) Stabilizing an unstable economy, Columbia University Press, USA.

Ros, J. (2001) "From the capital surge to the financial crisis and beyond: the Mexican economy in the 1990's" in Ffrench-Davis, R., ed., Financial crises in "successful" emerging economies, ECLAC-Brookings Institutions Press, USA, 107-140. 
Öcal, N. \& Osborn, D. (2000) "Business cycle non-linearities in UK consumption and production", Journal of Applied Econometrics, 15, 27-43.

Rand, J. \& Tarp, F. (2002) "Business cycles in developing countries: are they different?", World Development, 30, 12, 2071-88.

Sichel, D. (1994) "Inventories and the three phases of the business of the cycles", Journal of Business and Economics Statistics, 12, 269-277. 